\title{
Leprosy: clinical and epidemiological study in patients above 60 years in Espírito Santo State - Brazil ${ }^{*}$
}

\author{
Lucia Martins Diniz¹, Leonardo Bezerra Maciel²
}

DOI: http:/ / dx.doi.org/10.1590/abd1806-4841.20187092

\begin{abstract}
BACKGROUND: Leprosy is a chronic infectious disease that is endemic in Brazil and little studied in patients over 60 years old.

ОвлестіvE: The aim of this study was the epidemiological and clinical description of cases of leprosy in individuals older than 60 years, notified in the State of Espírito Santo - Brazil.

MetHods: It was an observational, descriptive and retrospective study of leprosy patients notified between 2001 and 2011.

Results: Out of 16,025 notifications, 2,510 (15.6\%) were of patients over 60 years of age; the distribution among the period was egalitarian except in the last 2 years, when there was a mild reduction of cases; the average was 70 years of age $( \pm 7$ years); $46 \%$ were women, being $62 \%$ of those paucibacillary cases; 1,145 (50.5\%) were dark-skined people in particular among paucibacillary cases; $1,638(72.9 \%)$ were illiterates or poorly instructed n particular among multibacillary cases $(P=0.022)$; $59.9 \%$ patients were clinically multibacillary cases and $37.4 \%$ had positive bacilloscopy; $37.9 \%$ patients had clinical alterations on peripheral nerves, $36.7 \%$ of all the multibacillary cases were classified as grade I and $15.3 \%$ as grade in assessment of disability.

StUDy LIMITATIONS: This was a retrospective study, which used secondary data generated by physicians and notified by other professionals, whar could have enabled possible errors on original data.

ConCLUSIONS: Leprosy in this age group suggests a long incubation period with reactivation of latent focus or late infection. Men were more affected, as well as the afro descendent race was statistically significant in the paucibacillary cases $(\mathrm{P}=0.000)$ and illiterate/poor education in multibacillary cases $(\mathrm{P}=0.022)$. Nearly $40 \%$ of patients had positive bacilloscopy and grade I/ II disability, demonstrating a late diagnosis.
\end{abstract}

Keywords: Aged; Epidemiology; Leprosy

\section{INTRODUCTION}

Leprosy is a chronic infectious disease that affects the skin, the peripheral nerves and sometimes internal organs, depending on the degree of dissemination of the Mycobacterium leprae, of the patient's immunological response towards the bacillus and genetic characteristics of the individuals. ${ }^{1}$

Life expectancy for the Brazilian population has increased over the years with the promotion of healthcare established by law with the Brazilian Constitution of 1988 and with the multiple governmental and non-governmental actions for the promotion of health of the population older than 60 years. ${ }^{2}$ Despite all this, leprosy in patients older than 60 years is discussed in conjunction with general clinical and epidemiological data of other leprosy cases, without specifically focusing on this age group.

According to IBGE's data (Brazilian Institute of Geography and Statistics), in 2011, Brazil had 23.5 million people over 60 years of age, with $8,964,850$ of those the ones responsible for the house-

\footnotetext{
Received 13 March 2017.

Accepted 31 July 2017.

* Work conducted at the Centro de Ciências da Saúde, Service of Dermatology, Universidade Federal do Espírito Santo, Vitória (ES), Brazil.

Financial support: None.

Conflict of interest: None.

Discipline of Dermatology, Department of Internal Medicine, Universidade Federal do Espírito Santo, Vitória (ES), Brazil.

Discipline of Dermatology, Universidade Federal do Maranhão, São Luís (MA), Brazil.
}

E-mail: 1diniz0304@hotmail.com 
hold, with a mean age of 69 years, mean schooling of 3 years and 4 months and mean monthly income of $\mathrm{R} \$ 657.00 .^{2}$

The transmission of leprosy depends on contact with multibacillary patient. ${ }^{1,3}$ Literature data show that in previously endemic countries (Norway and Japan), when there was a reduction in the detection rates of new cases, there was an increase in the percentage of multibacillary cases in older age groups, indicating reduction in transmission of the infection. ${ }^{4,5}$

It is important to analyze individuals older than 60 years because in the absence of a diagnosis both transmission and occasional sequelae can increase, besides the possibility of occurrence of other diseases and drug interactions between their treatment with leprosy treatment in the older age group. It has been previously shown, in Brazil, in a study on the patterns of mortality related to leprosy and its association with diabetes mellitus and systemic hypertension between 1999 and 2007, that most deaths occurred in males and individuals older than 70 years, And, the risk factors statistically significant associated to death in leprosy, as primary cause, were adverse events to drugs, toxic liver disease, lower limb ulcers, kidney failure and sepsis $(P=0.001){ }^{6}$

The aim of this study is to describe the epidemiology and clinical aspects of cases of leprosy in individuals older than 60 years who were notified to national database by the State of Espírito Santo, Brazil.

\section{METHODS}

Observational, descriptive and retrospective study of cases of leprosy notified to the Secretaria de Estado da Saúde do Espírito Santo (SESA/ES) through records of mandatory notification of Brazil's database from January 2001 to December 2011.

The records were filled out by professionals of the Family Health Program (FHP). The diagnoses were made by general practitioners and dermatologists from all 78 municipalities of the State of Espírito Santo.

The following data were recorded in the study: date of diagnosis, name of patient, age, gender, skin color, municipality of residence, clinical form of the disease, degree of disability, operational classification, bacilloscopy from intradermal scraping, number of thickened nerves and number of cutaneous lesions at the moment of diagnosis for the preparation of a SPSS Statistics 17.0 statistical program spreadsheet.

Data were tabulated and analyzed statistically, determining the frequencies and respective percentages. We adopted as baseline the significance level of $P \leq 0.05$.

The project was approved by the Committee of Ethics in Research of the Centro de Ciências da Saúde, Universidade Federal do Espírito Santo under the number 151/11 and of the Committee of Ethics in Research of the Hospital Infantil Nossa Senhora da Glória, Secretaria de Estado da Saúde do Espírito Santo, under the number 05/2012.

\section{RESULTS}

During the period of the study, 16,025 cases of leprosy were notified and patients older than 60 years represented $15.6 \%$ of the total 2,510 cases.
The number of cases per year had little variation, with a subtle decrease on the last 2 years (2010 and 2011), as shown in figure 1 .

The median age of this group was 69 years and the mean corresponded to 70 years (standard deviation of 7 years), being the minimum age 61 years and the maximum 102 years. Regarding age group, most cases were concentrated between 61 and 70 years, with $1,469(58.5 \%)$ cases, followed by the age groups between 71 and 80 years (809 cases/32.2\%), 81 and 90 years (197 cases /7.8\%) and lastly, between 91 and 102 years ( 35 cases $/ 1.4 \%$ ).

Regarding gender, 1,154 (46\%) of the cases were female and 1,356 (54\%) were male; 716 (62\%) females were paucibacillary cases and 438 (38\%), multibacillary cases. In men, 764 (56.3\%) cases were the paucibacillary forms and $592(43.7 \%)$, multibacillary. Being female was statistically significant, with $\mathrm{OR}=1.10(1.03-1.17 ; P=0.004)$, to develop paucibacillary forms.

Of the total of cases in the study, 2,264 records were filled out with the color of the skin. We observed that 1,085 (47.9\%) individuals were caucasians, 1,145 (50.5\%) were of mixed races/black and 34 (1.5\%) were Asians. Being of mixed races/black was statistically significant in the development of paucibacillary forms with $\mathrm{OR}=1.21$ (1.09-1.35) and $P=0.000$.

The level of schooling was low, represented by 1,638 (72.9\%) illiterate individuals or those who had an incomplete elementary grade level, of a total of 2,248 records with this information. The remaining 610 individuals (27\%) had completed the elementary grade up to university degree. No schooling or illiteracy was statistically significant for the multibacillary forms with $\mathrm{OR}=1.11$ (1.01-1.21) and $P=0.022$.

Subdividing the State of Espírito Santo into zones, we observed that from the total of 2,490 records completed, 1,323 (53\%) cases lived in the central region of the State, formed by Grande Vitória and the municipalities of Vitória, Serra, Cariacica and Vila Velha, and 996 (40\%) individuals lived in the north coast and northwest region of the State of Espírito Santo. The south region was represented 171 (6.9\%) individuals.

In the group studied, 2,469 records were completed with the clinical features of leprosy and 301 (12.2\%) individuals had the indeterminate form of the disease; 688 (27.9\%) individuals had the tuberculoid form; 907 (36.7\%) individuals had the borderline form; and $573(23.2 \%)$ individuals had the lepromatous form, meaning that $40.1 \%$ had paucibacillary forms and $59.9 \%$, multibacillary. To have the indeterminate and tuberculoid forms was statistically significant for paucibacillary forms $(P=0.000)$.

Of the 2,298 records completed for the item "number of cutaneous lesions", 391 (17\%) individuals did not have cutaneous lesions or there were doubts on how to answer this criteria; 1,192 (51.8\%) individuals had 1 to 5 lesions on the skin; and 715 (31.2\%) individuals had more than 5 cutaneous lesions. Paucibacillary patients with up to 5 cutaneous lesions were 749 (96.9\%), with statistical significance $(P=0.000)$ compared to $443(39.1 \%)$ from multibacillary individuals with up to 5 cutaneous lesions.

Of $852(33.9 \%)$ individuals who had their peripheral nerves investigated, 529 (62.1\%) did not show features of neural involvement and 323 (37.9\%) had thickening, pain or shocks related to in- 


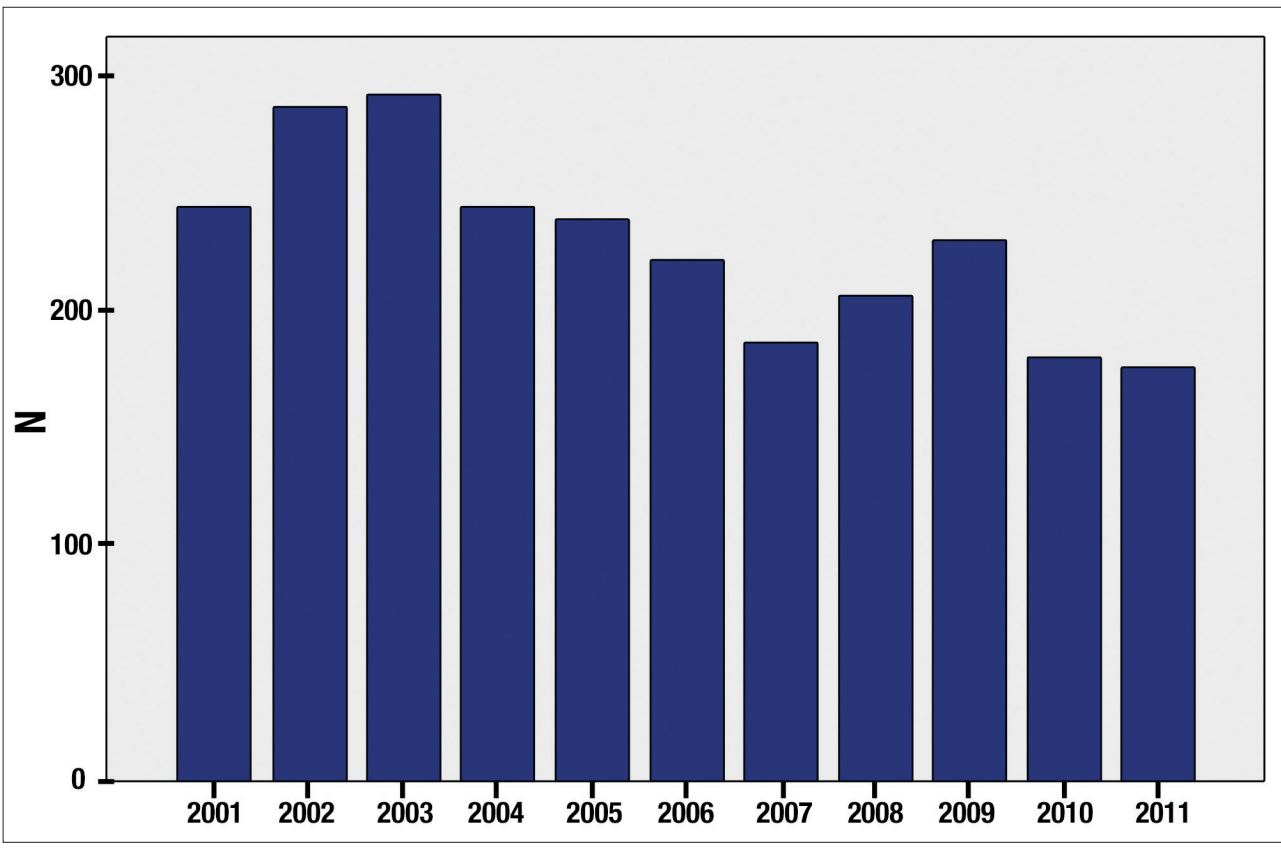

Year
FIGURE 1: Distribution of 2,510 patients older than 60 years with leprosy from 2001 to 2011 volvement of the peripheral nerves. There was no abnormality in the peripheral nerves in $243(76.9 \%)$ individuals with paucibacillary forms, statistically significant datum with $\mathrm{OR}=2.03$ (1.62-2.53) and $P=0.000$ compared to 286 (53.4\%) individuals with multibacillary forms who did not have any peripheral nerve abnormality either.

Regarding the assessment of the degree of disability, 2,298 individuals were evaluated and notified, with no disability in 1,381 $(60.1 \%)$ individuals; grade I disability was present in $657(28.6 \%)$ individuals and grade II affected 260 (11.3\%) individuals. Regarding paucibacillary forms, grade I disability was seen in 157 (16.8\%) individuals and grade II, in 51 (5.5\%) individuals. Regarding multibacillary forms, 500 (36.7\%) individuals were assessed as grade I and $209(15.3 \%)$ individuals had grade II.

Bacilloscopy of intradermal scraping was performed in $1,859(74 \%)$ individuals, being negative in 1,163 (62.6\%) individuals and positive in $696(37.4 \%)$. Regarding the operational classification, $13(1.3 \%)$ paucibacillary individuals had a positive bacilloscopy and $683(76.2 \%)$ multibacillary individuals had a positive test, which was statistically significant with $\mathrm{OR}=5.35$ (4.74-6.05) and $P=0.000$.

\section{DISCUSSION}

In this study, individuals older than 60 years represented $15.6 \%$ of leprosy patients during the 10-year period. Nobre et al. (2017) conducted an epidemiological study with the database of leprosy in Brazil from 2001 to 2013, in order to identify the populational groups that are more susceptible to develop multibacillary forms of the disease. Of the total of 541,090 cases analyzed, $17.5 \%$ were older than 60 years. ${ }^{7}$ Gomes et al. (2005) described 967 cases diagnosed with leprosy in a reference center in Ceará in 2004, with 117 (12\%) of them occurring in patients older than 65 years. ${ }^{8}$ Sousa et al. (2012), when studying 12,238 cases of leprosy notified between 2003 and 2008 in the database of the State of Piauí, observed that $19.4 \%$ were older than 60 years. ${ }^{9}$ Ninh (2014) found 23 cases $(18.2 \%$ ) of patients older than 60 years of a total of 126 cases of leprosy notified in Sarawak (Malaysia) between 2008 and 2012. ${ }^{10}$

In our study, we observed that in the 10-year period (2001 to 2011), there was a small reduction in the number of cases of leprosy in the last two years. Nobre et al. (2017), when analyzing the total of new cases diagnosed in all Brazilian states from 2001 to 2013, demonstrated a progressive reduction in the yearly detection rate of new cases, but a progressive increase in multibacillary cases in individuals older than 60 years, what could indicate a reduction of the transmission of the leprosy bacillus in Brazil. However, since this was also observed in states that are still hyperendemic, such as those in the North and Mid-West regions, the authors consider the possibility that this increase in multibacillary cases in older patients could be related to the higher life expectancy and lower immune response in those aged over 60 years. ${ }^{7}$ In the study by Larrea et al. (2012) about the pattern and trend of leprosy in Mexico between 1989 and 2009, there was a reduction of $80 \%$ in the number of detection of cases in people younger than 20 years and of $41 \%$ in patients older than 60 years. ${ }^{11}$ In the study by Lee et al., the reduction in the incidence of new cases of leprosy in Korea from 1977 to 2013 was due to better socioeconomic conditions of the population, allowing for improved personal and family hygiene, including the nutritional status, skin health and housing standards, which are contributing factors by themselves in the reduction of the transmission leprosy. ${ }^{12}$ The mean age of the individuals in this study was 70 years, 
confirming IBGE's data from 2012 in regard to the population older than 60 years. $^{2}$ The range was from 61 to 102 years, demonstrating the importance of full skin examination of the patients for the clinical detection of leprosy, even in old ages. In the study be Lee et al., with the reduction of new cases of leprosy in Korea, the mean age of the new cases diagnosed went from 40 to 65 years, denoting that those patients acquired the disease many years back, with multibacillary cases being more frequent in this age group, same as seen in this study, due to the long incubation period of the disease. ${ }^{12}$

In our study, most patients $(90.7 \%)$ were between 61 and 80 years of age, in accordance to IBGE's data (2010) regarding the most frequent age groups according to Brazilians' life expectancy. ${ }^{13}$ Ajalla et al. (Mato Grosso do Sul), when studying leprosy in the cities of Coronel Sapucaia and Paranhos, noticed the highest coefficient of detection in patients older than 70 years. ${ }^{14}$ Larrea et al. (2012) also observed an increase in the number of cases in patients older than 60 years, mainly between 60 and 79 years, raising the possibility of acquisition of the bacillus later in life or many years before with a long incubation period, what is consistent with the increase in the number of multibacillary cases in this age group. ${ }^{11}$ Basel et al. (2014) raised the possibility of reactivation of latent infections when leprosy occurs in older patients. ${ }^{15}$

In the IBGE 2010 census, it was identified that the population aged over 60 years in the State of Espírito Santo was of 364,745 individuals, with 200,218 (54.8\%) females. ${ }^{13}$ In our study, the leprosy in this age group was more frequent in males (54\%) compared to females $(46 \%)$, being paucibacillary cases more evident among females $(P=0.004)$. Castro et al. (2016) noticed a predominance of leprosy in males when compared to females in all Brazilian states in $2010 .{ }^{16}$ Nobre et al. (2017), in the analysis of 541,090 cases of leprosy in Brazil, also demonstrated that the number of cases in males was significantly higher than in females $(P=0.0001) .{ }^{7}$ Longo and Cunha (2006), when describing 192 patients with leprosy, observed that 34 $(17.7 \%)$ patients were older than 60 years, with $23(67.6 \%)$ cases in males. ${ }^{17}$ In the study by Cruz Silva et al. (2015), from the total of 1,916 new cases of leprosy in Juazeiro (Bahia), during the period of 2002 to 2012, 340 patients were older, with 182 (53.5\%) males and $158(46.5 \%)$ females. ${ }^{18}$

The State of Espírito Santo had $48.6 \%$ of the individuals self-declared brown-skinned in the census of $2010 .{ }^{13}$ The individuals in the study were more frequently brown/black-skinned, with paucibacillary cases statistically more frequent $(P=0.000)$. Regardless of age, Castro et al. (2016), in a study on the incidence of leprosy in Brazilian states, found a higher frequency of the disease in non-white patients, suggesting a higher exposure of these patients to social conditions that facilitate the transmission of the disease. ${ }^{16}$

According to IBGE's data (2012), the population above 60 years of age has a mean schooling of 3 years and 4 months, data in accordance with the majority of the cases $(72.9 \%)$ in the study, made of illiterate or those with incomplete elementary grade. ${ }^{2}$ However, poor schooling was statistically significant $(P=0.022)$ for multibacillary cases. In a study in Mato Grosso do Sul, patients with leprosy and illiterate or with less than 4 years of schooling represented $44.2 \%$, and, in the municipality of Sete Quedas, this group was $66.6 \% \cdot{ }^{14}$ Kerr-Pontes et al. (2006) suggest that poor poor schooling is one of the risk factors for transmission of leprosy $(\mathrm{OR}=2.05)$. This demonstrates that the lack of education favors precarious conditions of living, predisposing to the transmission of leprosy. ${ }^{19}$

The majority (93\%) of cases of leprosy in the study lived in the central, north and northwest regions of the State of Espírito Santo, an area considered as part of cluster 4, or clustering of leprosy cases, together with the northeast of Minas Gerais and south of Bahia, as demonstrated by the Department of Epidemiology of the Brazilian Ministry of Health in 2009 regarding the concentration of all cases of leprosy in the State. ${ }^{20}$

Of note, near $60 \%$ of the cases of leprosy in the study had the borderline and lepromatous forms. Even in the case of diagnostic uncertainty of the clinical forms, we can see that from the total of patients that underwent bacilloscopy of intradermal scraping, in $37.4 \%$ of the cases this test was positive, allowing for the transmission of the disease and, therefore, being diagnosed later. Nobre et al., when analyzing 541,090 notified cases of leprosy in Brazil from 2001 to 2013, described that individuals older than 60 years had double the cases of multibacillary disease compared to those younger. ${ }^{7} \mathrm{Li}$ et al. (2011), when studying the detection rate of leprosy in Yuxi, province the southeast China, between 1952 and 2008, described 2,223 cases, being $95(4.34 \%)$ in patients older than 60 years and, of those, $42(44.2 \%)$ patients were multibacillary. ${ }^{21}$ Sousa et al. observed an increase of $26 \%$ in multibacillary forms 2003 and 2008 in the State of Piauí, proportional to aging, reflecting the long incubation period of the disease or a lack in early diagnosis. ${ }^{9}$ The high number of multibacillary cases with a positive bacteriological index, can increase the risk of transmission of leprosy among non-immune persons. ${ }^{22}$

In the notification records, 391 (17\%) patients had no cutaneous lesions. This could be explained by a typing error, lack of appropriate completion of the form or cases with exclusive involvement of peripheral nerves. Most individuals (51.8\%) in the study had 1 to 5 skin lesions, seen in $96.9 \%$ of the paucibacillary cases, which was statistically significant $(P=0.000)$. More than 5 skin lesions were present in $691(60.9 \%)$ of the multibacillary cases, in disagreement with Gallo et al. (2003), when studying the clinical correlation of the number of cutaneous lesions and bacilloscopy results. The authors found $83.8 \%$ of the cases with up to 5 skin lesions with no bacilli in laboratory tests and, of the 584 cases with a positive test, $89.6 \%$ had more than 5 cutaneous lesions. ${ }^{23}$

Few cases in the study (33.9\%) had the peripheral nerves assessed, but $37.9 \%$ showed signs and/or symptoms of nerve involvement. Of the total 536 multibacillary cases assessed, $46.6 \%$ had abnormal peripheral nerves. Multibacillary cases have a higher susceptibility to peripheral nerve damage due to the presence of bacilli and leprosy reactions. Consequently, this predisposes to disability, being also associated to late diagnosis. ${ }^{24}$

In the study, grades I and II disability were found in 39.9\% of individuals, being 208 paucibacillary cases and 709 multibacillary cases. Grade II disability points to a late diagnosis and to a flaw in healthcare regarding the early search for new cases. ${ }^{14}$ Late diagnosis of leprosy indicates poor schooling, lack of adequate socioeconomic conditions, little knowledge of the disease by the population and lack of training on the disease among health professionals. ${ }^{19,25}$ In the study by Cruz Silva et al. (2015), in Juazeiro (Bahia), the logistic re- 
gression of the data to determine factors associated to the degree of disability showed a connection to being male $(P=0.001)$, being older than 45 years $(P=0.000)$ and multibacillary $(P=0.000)$, variables compatible with those of this study. ${ }^{18}$

The early detection and protection of those susceptible can reduce the transmission of the disease, lessening physical disabilities and the stigma of leprosy. ${ }^{26}$ Hence the importance of individually studying the Brazilian populations, with special attention to age groups (below 15 years of age, economically active population represented between 15 and 60 years and those older than 60 years), according to the circumstances of each region.

\section{REFERENCES}

1. Scollard DM, Adams LB, Gillis TP, Krahenbuhl JL, Turman RW, Williams DL. The continuing challenges of leprosy. Clin Microbiol Rev. 2006;19:338-81.

2. Sdh.gov.br [Internet]. Ministério da Justiça e Cidadania. Secretaria Especial de Direitos Humanos. Dados sobre 0 envelhecimento no Brasil [cited 2017 Jan 30]. Available from: www.sdh.gov.br/assuntos/pessoa.../dados.../ DadossobreoenvelhecimentonoBrasil.pdf.

3. Bratschi MW, Steinmann P, Wickenden A, Gillis TP. Current knowledge on Mycobacterium leprae transmission: a systematic literature review. Lepr Rev. 2015;86:142-55

4. Irgens LM. Epidemiological aspects and implications of the disappearance of leprosy from Norway; some factors contributing to the decline. Lepr Rev. 1981;52:147-65.

5. Koba A, Ishii N, Mori S, Fine PE. The decline of leprosy in Japan: patterns and trends 1964-2008. Lepr Rev. 2009;80:432-40.

6. Ramos AVA. Mortalidade relacionada à hanseníase e sua associação com diabetes mellitus e hipertensão arterial sistêmica na população brasileira, 19992007 [tese]. Fortaleza (CE): Universidade Federal do Ceará; 2011. 152p.

7. Nobre ML, Illarramend X, Dupnik KM, Hacker MA, Nery JA, Jerônimo SM, Sarnos EN. Multibacillary leprosy by population groups in Brazil: Lessons from an observational study. PLoS Negl Trop Dis. 2017;11:e0005364.

8. Gomes CCD, Pontes MAA, Gonçalves HS, Penna GO. Clinical and epidemiological profile of patients diagnosed with leprosy in a reference center in the northeast of Brazil. An Bras Dermatol. 2005;80:S283-8.

9. Sousa MWG, Silva DC, Carneiro LR, Almino MLB, Costa ALF. Perfil epidemiológico da hanseníase no estado do Piaú, período de 2003 a 2008. An Bras Dermatol. 2012:87:401-7.

10. NNinh LH. Leprosy in Sarawak, Borneo: a 5 year review from 2008 to 2012. Lepr Rev. 2014;85:332-5

11. Larrea MR, Carreño MC, Fine PE. Patterns and trends of leprosy in Mexico: 19892009. Lepr Rev. 2012;83:184-94.

12. Lee J, Kim JP, Nishikiori N, Fine PE. The decline of leprosy in the Republic of Korea patterns and trends 1977-2013. Lepr Rev. 2015;86:316-27.

13. ljsn.es.gov.br [Internet]. Instituto Jones dos Santos Neves. Distribuição populacional no Espírito Santo: resultados do Censo Demográfico 2010. IJSN. 2011; IV:1-11 [cited 2017 Jan 30]. Available from: http://www.ijsn.es.gov.br/ ConteudoDigital/20121022_958_201127_.pdf.

\section{CONCLUSION}

The study of leprosy in individuals older than 60 years from 2001 to 2011 notified by the State of Espírito Santo concluded that 2,510 cases of leprosy were notified, representing $15.6 \%$ of all notified; the mean age of cases was of 70 years ( \pm 7 years), ranging from 61 to 102 years; males (54\%) were more frequently affected; brown/ black-skinned individuals were $50.5 \%$ of the cases, which was statistically significant $(P=0.000)$ for paucibacillary cases; those who were illiterate or had poor schooling represented $72.9 \%$, being a significant datum for multibacillary cases $(P=0.022)$; there were more multibacillary cases (60\%); $51.8 \%$ of the cases had between 1 and 5 lesions, being $96.9 \%$ of paucibacillary cases $(P=0.000)$; intradermal bacilloscopy was positive in $37.4 \%$ indicating a late diagnosis and assessment of the degree of disability in multibacillary cases identified $36.7 \%$ with grade I and $15.3 \%$ with grade II. $\square$

14. Ajalla ME, Oliveira De Andrade SM, Tamaki EM, Waissmann W, Deittrich SH, Aragão Do Nascimento V. Leprosy in Brazilian counties bordering Paraguay: Mato Grosso do Sul State, 2001-2011. Lepr Rev. 2016;87:32-41.

15. Basel P, Pahan D, Moet FJ, Oskam L, Richardus JH. Leprosy incidence: six years follow-up of a population cohort in Bangladesh. Lepr Rer. 2014;85:158-69.

16. Castro SS, Santos JP, Abreu GB, Oliveira VR, Fernandes LF. Leprosy incidence characterization of cases and correlation with household and cases variables of the Brazilian states in 2010. An Bras Dermatol. 2016;91:28-33.

17. Longo JDM; Cunha RV. Perfil clínico-epidemiológico dos casos de hanseníase atendidos no Hospital Universitário em Campo Grande, Mato Grosso do Sul, de janeiro de 1994 a julho de 2005. Hansen Int. 2006;31:9-14.

18. Cruz Silva MEG, Souza CDF, Silva SPC, Costa FM, Carmo RF. Epidemiological aspects of leprosy in Juazeiro-BA, from 2002 to 2012. An Bras Dermatol. 2015;90:799-805.

19. Kerr-Pontes LR, Barreto ML, Evangelista CM, Rodrigues LC, Heukelbach J, Feldmeier H. Socioeconomic, environmental, and behavioural risk factors for leprosy in North-east Brazil: results of a case-control study. Int J Epidemiol 2006;35:994-1000.

20. Brasil. Ministério da Saúde. Secretaria de Vigilância em Saúde. Departamento de Vigilância Espidemiológica. Hanseníase no Brasil: dados e indicadores selecionados. Brasília: Ministério da Saúde;2009.

21. Li YY, LiXA, He L, Wang D, Chen WY, Chen L. Trends in new leprosy case detection over 57 years (1952-2008) in Yuxi, Yunnan Province of Southwest China. Lepr Rev. 2011;82:6-16.

22. Shen J, Zhou M, Xu X, Ray A. Zhang G, Yan L. A big challenge in case finding at low endemic situation: Analysis on 1462 new leprosy patients detected in China in 2007. Lepr Rev. 2010;81:176-83.

23. Gallo MEN, Ramos Júnior LAN, Albuquerque ECA, Nery JAC, Sales AM. Alocação do paciente hanseniano na poliquimioterapia: correlação da classificação baseada no número de lesões cutâneas com os exames baciloscópicos. An Bras Dermatol. 2003;78:415-24.

24. Alencar CH, Ramos AN Jr, Barbosa JC, Kerr LR, De Oliveira ML, Heukelbach J. Persisting leprosy transmission despite increased control measures in an endemic cluster in Brazil: the unfinished agenda. Lepr Rev. 2012;83:344-53.

25. Brito AL, Monteiro LD, Ramos Junior AN, Heukelbach J, Alencar CH. Temporal trends of leprosy in a Brazilian state capital in Northeast Brazil: epidemiology and analysis by joinpoints, 2001 to 2012. Rev Bras Epidemiol. 2016;19:194-204.

26. El-Dawela RE, Mohamed AS, Yousef F. Analysis of newly detected leprosy in Sohag Governorate, Upper Egypt, 2004 - 2008. Lepr Rev. 2012;83:71-9.

\footnotetext{
AUTHORS'CONTRIBUTIONS

Lucia Martins Diniz

Elaboration and writing of the manuscript

Leonardo Bezerra Maciel

(iD) ORCID 0000-0001-8107-8878

iD ORCID 0000-0002-4380-379X

Approval of the final version of the manuscript
}

How to cite this article: Diniz LM, Maciel LB. Leprosy: clinical and epidemiological study in patients above 60 years in Espírito Santo State Brazil. An Bras Dermatol. 2018;93(6):824-8. 